\title{
ESQUEMAS CONCEPTUALES PREVIOS DE LOS ALUMNOS EN GEOLOGÍA
}

GRANDA VERA, A.

I.B. Pedro Espinosa. Antequera (Málaga).

\section{SUMMARY}

The presence of conceptual errors or pre-scientific ideas about Geology in our students runs parallel to what they sustain about other sciences: we observe not only their persistence but also their similarity to concepts/theories from previous scientific periods.

\section{INTRODUCCIÓN}

Las investigaciones didácticas sobre los procesos de enseñanza/aprendizaje en Geología están poco desarrolladas; parece que la didáctica de esta ciencia, al menos en nuestro país está en sus comienzos, en to que se refiere a los nuevos paradigmas psicológicos y pedagógicos. Del análisis bibliométrico de las publicaciones sobre ciencias experimentales realizadas en 1985, confeccionado por Mata y Anta (1986) se desprende que de los 205 trabajos reseñados sólo 8 corresponden a Didáctica de la Geología, si excluimos los de carácter general y los medio ambientales; este dato es más revelador si consideramos que «frente al predominio tradicional de los trabajos sobre Fisica y Química en el período 1975-1983 se confirma un cambio en la tendencia, con la equiparación -producida ya en el 1984- de la producción sobre Ciencias Naturales (en torno al $48 \%$ del total) con respecto a las de Fisica y Quimica (aproximadamente el $42 \%$ )" (Mata y Anta, 1986). En suma tal equilibrio se produce por el avance de los estudios en Biologia e investigación del medio básicamente.

Aunque estamos de acuerdo en la especificidad de cada ciencia en los aspectos que afectan a su Epistemología y su Didáctica (Shayer y Adey 1984), no es menos cierto que comparten elementos comunes, al igual que en el plano científico estricto, así se detectan en Geologia, ideas y esquemas previos en los alumnos, sustentados en observaciones y conclusiones de sentido común. Estas barreras para conseguir un aprendizaje significativo se ven aumentadas por las dificultades para comprender la escala del tiempo geológico, la dimen- sión de los cambios, el detallismo de muchos fenómenos geológicos, las relaciones profundas entre procesos corticales e internos, etc... sin olvidar la complejidad, e incluso imposibilidad de diseñar experiencias realizables, y por tanto la necesidad de formular modelos analógicos correctos.

Algunos de los obstáculos expuestos son compartidos por la Biologia, al igual que la necesidad de matizar e incluso combatir las noticias excesivamente simples de los medios de comunicación de masas.

El trabajo que se presenta a continuación se ha realizado para iniciar una investigación acerca de la validez o no de estas ideas en el campo de la Didáctica de la Geologia. Los cuestionarios y observaciones se han dirigido a tres grupos de $3^{\circ}$ de BUP durante dos cursos consecutivos, del I.B. "Pedro Espinosa» de Antequera; a los citados grupos se les impartió una programación experimental de la asignatura de Ciencias $\mathrm{Na}$ turales de ese curso, aprobada por la Consejería de Educación y Ciencia de la Junta de Andalucía. No se pretende agotar Ia relación de errores conceptuales, ni las hipótesis de su aparición, sino contribuir a abrir líneas de investigación en tal sentido o a continuarlas.

\section{ESQUEMAS CONCEPTUALES E IDEAS PREVIAS DE LOS ALUMNOS}

Los alumnos escogen aquellos datos y observaciones que les son significativos en el marco de referencia de las teorias que aceptan y creen; asi en multitud de oca- 
siones los profesores comentamos que permanecen como relevantes la anécdota o el ejemplo marginal, teriendo su correlato en las respuestas a pruebas o exámenes.

$\mathrm{Si}$ aceptamos que los conceptos cambian de valor (de significado), ai variar el esquema conceptual en que se hallan inmersos, comprenderemos la importancia de generar cambios de marcos conceptuales mediante las metodologias adecuadas. Previamente hay que desvelar sobre qué se apoyan los alumnos, qué cargas teóricas impregnan a los hechos (Brown 1984), en suma, mantener una actitud experimental, partiendo de las preguntas y representaciones de los alumnos (Giordan 1985a).

Una de las vias actuales de investigación didáctica la constituye la detección de los errores conceptuales, ideas previas o preconceptos cientificos (Giordan 1985b), (Astudillo y Gené 1984), (Gené 1982), (CabaIler 1985), (Jiménez 1985), (Pedrinazi 1987).

Las características de dichos preconceptos son, según Solís Villa (1984) citando a Osborne y Wittrock, las siguientes:

- no son congruentes con los conceptos, leyes y teorías que los alumnos tienen que aprender.

- constituyen un esquema conceptual coherente, con amplio poder explicativo.

- son muy resistentes al cambio; a veces no cambian en absoluto; cuando se produce el cambio, como resultado del estudio, puede no coincidir con lo previsto por el profesor.

- interfieren en el aprendizaje de las ciencias, siendo responsables de la dificultad que encuentran los alumnos en estas asignaturas.

Driver (1986) insiste en las relaciones que se establecen entre tales ideas o preconceptos en el campo delimitado por los esquemas conceptuales alternativos de los estudiantes; además de las características enumeradas anteriormente señala:

- los estudiantes manejan un lenguaje impreciso y términos indiferenciados para expresar sus ideas.

- ideas intuitivas similares son detectadas en estudiantes de diferentes medios y edades.

Por último hemos de señalar el paralelismo (que no la copia exacta), entre los preconceptos y su evolución, y los cambios conceptuales que se han producido en el transcurso de la historia de la ciencia (Pjaget 1970).

\section{LOS PROBLEMAS METODOLÓGICOS}

Nos encontramos con otras dificultades, que sólo in dicaré brevemente, como la tendencia, de profesores y alumnos, a la respuesta rápida y superficial a los interrogantes que nos plantea la naturaleza (Carrascosa y Gil 1985), acrecentada por la densidad enciclopédica de los temarios (que llega al extremo en el COU), y por el escaso interés de los alumnos en profundizar sobre las cuestiones que se les plantean, por creer que tienen la contestación adecuada; a su vez las preguntas que ellos se pueden formular repiten los tópicos de los medios de comunicación de masas (Giordan 1985a). Estos problemas y los expuestos en el apartado anterior han tratado de ser superados con las propuestas de programación experimental de Ciencias Naturales para $3^{\circ}$ de BUP que se han llevado a cabo los cursos $85 / 86$ y 86/87; una de las directrices básicas de éstas era conocer lo que pensaban los alumnos, con el fin de diseñar estrategias didácticas adecuadas para favorecer la construcción de su propio conocimiento.

\section{METODOLOGÍA EMPLEADA EN LA DE- TECCION DE LOS ERRORES CONCEPTUA- LES EN GEOLOGÍA}

Se seleccionaron preguntas sobre aquellas cuestiones que habian aparecido en respuestas a pruebas y ejercicios con un carácter repetitivo, y que avalaban una hipótesis de error conceptual; se pasaron cuestionarios sobre las mismas a los grupos que seguían la programación experimental efectuando una segunda selección más restringida para los alumnos del siguiente curso; las que se mantuvieron no se alteraron en su formulación. También se utilizó la observación y el debate entre los grupos de alumnos, pues se combinaba el trabajo individual y el colectivo en el curso experimental ya comentado.

A posteriori se han agrupado los posibles errores en diferentes bloques, al mismo tiempo que se ofrecen los resultados de las respuestas a cada cuestión, y un comentario en el que se postulan los posibles paralelismos con situaciones anteriores históricas de la Geologia.

\section{CLASIFICACIÓN DE LOS PRECONCEP- TOS EN GEOLOGIA}

I. Un primer grupo lo constituirian aquellos que se asemejan a teorías y explicaciones que se presentaron en fases anteriores del pensamiento científico geológico; en este grupo se encontrarian:

I.a. Relación núcleo incandescente-vulcanismo.

I.b. Confusión entre magnetismo y gravedad.

I.c. Movimientos del nivel del mat para explicar la aparición de fósiles a gran altura.

II. El «sentido común» dicta las respuestas a interrogantes que se planteen; podriamos decir que lo aparente subyuga, como en los casos que siguen:

II.a. Un granito se erosiona siempre menos que una caliza. (El Torcal para alumnos de Antequera está formado por granito $(10 \%)$ o rocas metamórficas $(22 \%)$. II.b. Relaciones causa -efecto entre sismicidad, vulcanismo y tectónica de placas. 
II.c. Confusión de rigidez con densidad.

III. El tercer grupo lo formarian las ideas previas, que se derivan de una falta de dominio de la metodología científica; los alumnos creen:

III.a. Que el gradiente geotérmico es un método.

III.b. Que las ondas sísmicas son sólo un método.

\section{CUESTIONES PLANTEADAS, ANÁLISIS Y COMENTARIOS SOBRE LAS RESPUESTAS}

I.a. ¿Qué pruebas aportarian para demostrar que hay un núcleo semifluido e incandescente en el interior de la tierra?

$69 \%$ los materiales que arrojan los volcanes

$12 \%$ el propio origen de la tierra, cuando era una

bola incandescente

$12 \%$ por la trayectoria de las ondas sísmicas al llegar al núcleo

$3 \%$ por las perforaciones que se realizan

$4 \%$ otras razones.

La respuesta mayoritaria presupone una comunicación directa entre el núcleo y el exterior terrestre, y por tanto la existencia de canales o zonas de contacto entre los focos volcánicos (nucleares) y la corteza; observamos además la inadecuada relación que se establece entre vulcanismo y tectónica global.

La explicación dada a la actividad volcánica y las teorías acerca del interior de la tierra hasta el siglo XVIII, incluso el XIX, coinciden con lo manifestado por la mayoría de nuestros alumnos. El paradigma de esta concepción figura en el libro de Atanasius Kircher, Mundus subterraneus; en él se describe y aparece dibujado un esquema de funcionamiento de la tierra en la que hay un gran fuego central interno y una red de canales para fuegos, aguas, éteres, etc. Buffon es de los primeros científicos que señala: «el fuego de los volcanes no procede de un fuego central, ni siquiera de un fuego muy profundo, pues el aire es absolutamente necesario para que se produzca o por lo menos para alimentarlo» (Taton 1972); las razones argüidas no son correctas, pero son coherentes con los conocimientos físico-quimicos y geológicos de la época.

I.b. ¿A qué factor/es se debe la existencia del campo gravitatorio terrestre? ¿Y el campo magnético?

$11 \%$ hacen responsable de la existencia de los dos campos a las mismas razones: movimientos del núcleo, tamaño, densidad, etc... explican el campo magnético por:

$17 \%$ el rozamiento entre capas del interior terrestre $9 \%$ la composición de $\mathrm{Fe}$ y $\mathrm{Ni}$ del núcleo

$6 \%$ la rotación de la tierra

$9 \%$ los polos terrestres que generan un imán

$48 \%$ no sabe/no contesta

explican la existencia de un campo gravitatorio por:

$59 \%$ la propia masa que es la tierra
$1 \%$ atracción del núcleo
$29 \%$ no sabe/no contesta.

Según Bernal (1979), Gilbert «no se limitó a los experimentos: esbozó a partir de ellos nuevas ideas generales. La que sorprendió más a la imaginación đe la época fue que los planetas se mantenían en sus órbitas gracias a la virtud magnética de la atracción». Dampier (1986) dice: «inspirándose (Gilbert) en la idea filosófica griega sobre un influjo etéreo e inmaterial, imaginó que el imán o sustancia electrificada emitía una especie de afluvio con el que cautiva los cuerpos circundantes y los atrae hacia sí; este mismo concepto lo aplicó a la explicación de la gravedad, es decir, a la fuerza que impulsa las piedras al suelon. En ambas citas se expresan las imprecisiones originales entre magnetismo y gravedad, confusiones que manifiestan algunos de los estudiantes, después de haber recibido una asignatura de Física y Química en $2^{\circ}$ de BUP; parece desprenderse un mejor conocimiento de la gravedad que del magnetismo. Historicamente hay que resaltar que algunas de las ideas de Gilbert sirvieron a Newton para formular sus teorias acerca de la gravitación.

I.c. ¿A qué altura estaba el nivel del mar en el momento de depositarse lo que actualmente son fósiles, respecto del nivel actual? (p. ej. en El Torcal de Antequera, con una altura media de unos mil metros)

$37 \%$ llegaba hasta las cimas actuales

sobrepasándolas

$47 \%$ más o menos como el actual (de ellos un 33\% indica que eran los materiales los que se habian levantado)

$12 \%$ a menos de mil metros

$4 \%$ no sabe/no contesta.

Se constata que, para explicar la presencia de fósiles, más de un $50 \%$ de los alumnos que han recibido una enseñanza de Ciencias Naturales en $1^{\circ}$ de BUP no indican por error u omisión que los materiales que forman las montañas y cordilleras se han levantado (se han movido), y lo que quizás sea más significativo es que casi la mitad de los encuestados tiene la idea arraigada de que han sido movimientos (enormes) del mar los responsables de tales estructuras geológicas. Es sorprendente la similitud de estos planteamientos con los que sostenían los medievalistas; Leonardo da Vinci los criticaba aduciendo que «los fósiles que se encuentran ahora en las altas montañas continentales se produjeron en el agua de mar y no se puede suponer que hubiesen llegado al punto en que se hallan hoy, en los cuarenta dias que duró el diluvio de Noé. En realidad ni juntas todas las aguas de tierra, nubes, ríos y océanos podrian llegar a cubrir las cordilleras de nuestro globo". Han tenido que producirse cambios en la corteza de la tierra, decía Leonardo; pero no hace falta recurrir a ningún cataclismo. «... con el tiempo, el Po se Ilevará la tierra seca al fondo del Adriático, de la misma forma que ya acarreó alli gran parte de la Lombardía" (Dampier 1986). 
Todavía Maiłlet en el siglo XVIII afirmaba: «os animales y vegetales terrestres son descendientes de los que vivían en el mar universal, cuya retirada originaria la emersión de las montañas». (Taton 1972).

II.a. Confusión entre resistencia a la erosión y dureza en su acepción común.

Antes de responder a la cuestión, los alumnos debatieron el comportamiento de materiales formados a condiciones de $\mathrm{P}$ y $\mathrm{T}^{\mathrm{a}}$ lejanas a las condiciones de la corteza, frente al de otros originados en medios similares o no muy lejanos del ambiente de la corteza, la mayoría llegaron a la conclusión de que los primeros serían más inestables, porque su estructura molecular estaba de acuerdo con las condiciones de formación; posteriormente se les preguntó: ¿Qué roca es más fácilmente erosionable a la $\mathrm{P}$ y la $T^{\mathrm{a}}$ de la corteza, un granito o una caliza? ¿Por qué?

$82 \%$ la caliza porque:

$15 \%$ sus enlaces químicos son más débiles

$30 \%$ es más blanda

$18 \%$ es menos compacta

$19 \%$ otras: más porosa, menos densa, más heterogénea, etc.

$9 \%$ el granito porque se forma a condiciones más alejadas de las dominantes en la corteza, que la caliza.

$9 \%$ no sabe/no contesta.

En las respuestas dadas para apoyar la hipótesis de las calizas se mezclan conceptos como la densidad, la blandura, la porosidad, etc... todos ellos, a mi entender, sinónimos de una idea vaga de dureza, que definiría la cualiad esencial que explica la resistencia a los agentes erosivos; la alteración química de un trozo «duro" de hierro puede invocarse como ejemplo, pero se comprueba entonces que para una gran parte de los estudiantes, la erosión es un concepto esencialmente mecánico siendo considerada por muy pocos las alteraciones de origen químico.

II.b. La cuestión se enfocó para conocer el grado de adquisición del concepto de causalidad (Giordan 1985a) en la tectónica global, sobre los aspectos más conocidos por los alumnos: volcanes y terremotos.

Los volcanes y terremotos, ison causa o efecto de los movimientos de las placas? Explica según los casos las razones que creas convenientes.
$3 \%$ son causa y efecto
$6 \%$ cuando se acercan las placas producen los te- rremotos; cuando se alejan, los volcanes.
$3 \%$ son la causa del movimiento de las placas
$6 \%$ los volcanes son causa porque expulsan lava; los terremotos efecto
$70 \%$ en el choque de placas se provocan los volca- nes y sismos
$12 \%$ no sabe/no contesta

Del análisis de las respuestas parece desprenderse que:
- el acercamiento de placas implica movimientos, desequilibrios, en suma sismicidad.

- el alejamiento de placas litosféricas hace pensar en agujeros, fosas, espacios abiertos por los que fluye el magma, es decir vulcanismo.

- un $\%$ elevado de alumnos cercano al 30 , tiene ideas muy confusas incluso incorrectas respecto al concepto de causalidad que se ha investigado.

- aún en el caso de los alumnos que dominan tal concepto, el choque de placas aparece como un ejemplo más creible, por lo que implica de catástrofe, cambios bruscos, etc... en relación con el vulcanismo y la sismicidad.

II.c. Confusión entre los conceptos de densidad y rigidez: consecuencias para un correcto aprendizaje del interior terrestre.

Las cuestiones planteadas fueron dos:

- ¿Qué factores influyen en la velocidad de las ondas sísmicas en su transmisión por el interior de la tierra?

$19 \%$ la composición de materiales

$34 \%$ la densidad

$16 \%$ el estado físico de las capas de la tierra

$6 \%$ profundidad de los materiales

$13 \%$ la presión

$12 \%$ la rigidez

\section{- Define lo que es rigidez}

$39 \%$ la existencia de fuerzas moleculares y enlaces fuertes que impiden las deformaciones

$24 \%$ dureza

$12 \%$ medio compacto por la presión

$9 \%$ depende de la densidad

16\% otros: no se doblan, no se rompen fácilmente, no elásticos, etc...

De las respuestas a la primera cuestión se deduce la importancia concedida al factor densidad, aunque se citan todos los importantes; de la rigidez, la mayoria de los estudiantes tiene una vaga noción que sobre todo se expresa en función de otros conceptos más conocidos por ellos. Estos hechos condicionan la comprensión de los motivos por los que se producen las variaciones de la velocidad provocando aparentes paradojas; habria que insistir en el sentido físico de la densidad como concepto que se refiere a la masa de los átomos, diferenciándola de la rigidez que informa de la flexibilidad de los enlaces atómicos, de las distancias entre los mismos, etc...; la densidad supone la inercia del medio a la propagación del movimiento ondulatorio, es por tanto un factor que influye de forma inversa en la velocidad de propagación.

Las constantes de la rigidez para cada medio físico son características, hasta llegar a la capa semifluida del núcleo en que la rigidez es cero; el coeficiente $\mu=\mathrm{O}$ y sin embargo $\rho$ ha aumentado a $9,5 \mathrm{gr} / \mathrm{cm}^{3}$. Las velocidades de propagación de las ondas sísmicas vienen 
determinadas por las fórmulas:

$$
v_{p}=\sqrt{\frac{k+\frac{4}{3} \mu}{\rho}} \quad v_{s}=\sqrt{\frac{\mu}{\rho}}
$$

donde $\mathrm{K}=$ módulo de comprensibilidad, $\mu=$ módulo de rigidez y $\rho=$ densidad (Lillo 1982).

III. a y b.

Las ondas sísmicas son ante todo un «método» para conocer el interior terrestre, antes que un fenómeno geológico con explicación basada en procesos físicoquímicos; así de la afirmación «las ondas $\mathrm{S}$ no se transmiten en medios líquidos o semiffuidos», los alumnos leen $\mathrm{e}$ interpretan "se envian ondas $\mathrm{S}$ porque éstas no se propagan en medios líquidos». Resulta difícil que los alumnos aprendan que del comportamiento de las ondas hemos obtenido datos indirectos del interior de la tierra.

El gradiente geotérmico para un $15 \%$ de los alumnos es un «método» y no un dato físico que nos permite deducir la existencia de estructuras en el interior que generan calor por diferentes mecanismos.

\section{CONCLUSIONES}

Los alumnos tienen que superar en el aprendizaje de la Geologia de $3^{\circ}$ de BUP, una serie de obstáculos epistemológicos originados por diferentes causas:

a) la presencia de errores conceptuales o preconceptos cientificos.

b) la confusión entre procesos y métodos, entre fenómenos y aplicaciones.

c) la influencia de un cierto "sentido común» y la aplicación de conceptos de la vida cotidiana.

d) la deficiente base conceptual físico-química.

e) la tendencia a olvidar los fenómenos y procesos internos, que no se ven y que necesitan esquemas conceptuales más abstractos y complejos para comprenderlos.

f) la dimensión de los conceptos de espacio y tiempo en Geologia.

g) una concepción básicamente estática del mundo y la naturaleza.

\section{REFERENCIAS BIBLIOGRÁFICAS}

ÁGUEDA, J. y ANGUITA, 1977, Geología. (Rueda: Madrid).

ASTUDILLO, H. y GENÉ, A.M., 1984, Errores conceptuales en Biologia: la fotosintesis de las plantas verdes, $E_{n-}$ señanza de las Ciencias, Vol 2, pp. 15-17.

BERNAL, J.D., 1979, Historia social de la Ciencia. (Península: Barcelona).

BROWN, H., 1984, La nueva filosofía de la ciencia. (Tecnos: Madrid).

CABALLER, $M^{2}$. J., 1985, Detección de preconceptos: una experiencia realizada en el primer curso de Ciencias Naturales de BUP, Enseñanza de las Ciencias, Vol, extra, pp. 36.

CARRASCOSA, J. y GIL, D., 1985, La metodologia de la superficialidad y el aprendizaje de las ciencias, Enseñan$z a$ de las Ciencias, Vol. 3, pp. 113-121.

DAMPIER, W.C., 1986, Historia de la Ciencia y sus relaciones con la filosofía y la religión. (Tecnos: Madrid).

DRIVER, R., 1986, Psicología cognoscitiva y esquemas conceptuales de los alumnos, Enseñanza de las Ciencias. Vol. 4, pp. 3-16.

GENÊ, A., 1982, La enseñanza de la Biología. Situación actual. Trabajo basado en la tesis de licenciatura: Els treballs pràctics en Biologia i la metodologia cientifica. Universitat de Barcelona.
GIL, D., 1983, Tres paradigmas básicos en la enseñarza de las ciencias, Enseñanza de las Ciencias. Vol. 1, pp. 26-34.

GIORDAN, A., 1985a, La Enseñanza de las Ciencias. (Siglo XXI: Madrid).

GIORDAN, A., 1985b, Interés didáctico de los errores de los alumnos, Enseñanza de las Ciencias, Vol. 2, pp. 11-18.

JIMÉNEZ ALEIXANDRE, $M^{2}$.P., 1985, Modelo de comportamiento de materiales ante fuerzas mecánicas, Enseñanza de las Ciencias, 3 (2), 108.113.

LILLO, J., 1982, Geologia. (Ecir: Valencia).

MATA, A. y ANTA, C., 1986, Las orientaciones actuales de la didáctica de las ciencias experimentales en Espanta. Enseñanza de las Ciencias. Vol 4, pp. 233-247.

PIAGET, J., 1970, La epistemologia genética. (A. Redondo: Barcelona).

PEDRINACI, E., 1987, Representaciones sobre los cambios geológicos. Investigación en la escuela. 2, 65-74.

SHAYER, M. y ADEY, P.H., 1986, La Ciencia de enseñar Ciencias. (Narcea: Madrid).

SOLÍS VILLA, R., 1984, Ideas intuitivas y aprendizaje de las ciencias. Enseñanza de las Ciencias. Vol. 2, pp. 83-91.

TATON, R. y otros, 1972, Historia general de la Ciencia. La Ciencia moderna. Vol. II, (Destino: Barcelona). 